\title{
Remark on "Indication, from Pioneer 10/11, Galileo, and Ulysses Data, of an Apparent Anomalous, Weak, Long-Range Acceleration"
}

\author{
Rainer W. Kühne \\ Fachbereich Physik, Universität Wuppertal, 42097 Wuppertal, Germany, kuehne@theorie.physik.uni-wuppertal.de
}

PACS numbers: 04.80.-y, 95.10.Eg, 95.55Pe

Recently, Anderson et al. [1] presented possible evidence for an apparent "anomalous acceleration" acting on the spacecrafts Pioneer 10/11 and, with less statistical significance, also on the spacecrafts Galileo and Ulysses.

The anomalous acceleration acting on these spacecrafts is reported to be $\sim 8 \times 10^{-8} \mathrm{~cm} / \mathrm{s}^{2}$, whereas the upper limit of that acting on the orbital motion of the planets Earth and Mars is $0.1 \times 10^{-8} \mathrm{~cm} / \mathrm{s}^{2}$.

This statement is incomplete, because it gives the (wrong) impression that an anomalous acceleration acting on planets and other large objects in the solar system is yet unknown. By contrast, the motions of the planets Uranus, Neptune, and Pluto show unexplained residuals. Furthermore, the motions of a number of comets (including the comets Halley, Encke, Giacobini-Zinner, and Borelli) are disturbed by an unknown origin.

A number of investigators proposed a non- gravitational force to explain the observed anomalous accelerations acting on comets [2]. Such a force, however, can hardly be accounted for the spacecraft data, because, as recognized by Anderson et al. [1], it would either violate the general relativistic equivalence principle many orders above the experimental upper limit or not explain the apparent independence of the anomalous acceleration on the distance from the Sun.

Other investigators suggested a transplutonian planet for the explanation of the anomalous cometary and planetary accelerations [3. Its mass was predicted to be of the order of that of the planet Saturn. However, such a large planet is unlikely to have escaped discovery by optical and infrared (especially IRAS) searches.

By contrast, a transneptunian comet or asteroid belt (14 cannot yet be excluded. Further examinations are required to show whether this hypothetical belt (gravitational effects; resistance by dust particles) can be accounted for both the disturbed motions of planets and comets and the possible anomalous accelerations acting on spacecrafts.

[1] J. D. Anderson et al., gr-qc/9808081 (to be published); a possible conventional explanation for the "anomalous acceleration" was suggested by J. I. Katz, gr-qc/9809070.

[2] F. L. Whipple, Astrophys. J. 111, 375 (1950); B. G. Marsden, Astron. J. 74, 720 (1969), ibid. 75, 75 (1970), ibid. 76, 1135 (1971); D. K. Yeomans, Astron. J. 76, 83 (1971); T. Kiang, Mon. Not. R. Astron. Soc. 162, 271p (1973).

[3] K. H. Schütte, Popular Astron. 57, 176 (1949); R. A. Naef, Orion 4, 484 (1955); J. E. Gunn, New Sci. 48, 345 (1970); W. D. Rawlins, Astron. J. 75, 856 (1970); P. K. Seidelmann, Astron. J. 76, 740 (1971); J. L. Brady, Publ. Astron. Soc. Pac. 84, 314 (1972); W. D. Rawlins and M. Hammerton, Mon. Not. R. Astron. Soc. 162, 261p (1973).

[4] F. L. Whipple, Proc. Nat. Acad. Sci. USA 51, 711 (1964). 